\title{
Surface Fatigue Crack Growth Simulation Based on the Finite-Element Aided Damage Accumulation Procedure
}

\author{
Ruslan V. Guchinsky*a,b and Sergey V. Petinova,b \\ ${ }^{a}$ Institute for Problems of Mechanical Engineering RAS \\ 61 V.O., Bolshoj pr., St. Petersburg, 199178, Russia \\ ${ }^{b}$ Peter the Great St. Petersburg Polytechnic University \\ 29 Polytechnicheskaya Str., St. Petersburg, 195251, Russia
}

Received 07.04.2015, received in revised form 12.09.2015, accepted 26.10.2015

\begin{abstract}
Presented is an approach addressed to evaluation of the surface and three-dimensional fatigue crack growth based on finite-element aided modeling of the damage accumulation. The approach incorporating the Strain-life criterion for fatigue failure and lumped cyclic stress-strain curve of material, may be advantageous compared to currently employed linear elastic fracture mechanics. It provides fatigue analysis without subdivision into the crack initiation and propagation; it allows naturally considering material plasticity and the crack opening effect. The approach application is illustrated in example of fatigue crack extensions from circular hole in a cyclically loaded plate. The results of simulation are in good agreement with experimental data.
\end{abstract}

Keywords: quarter-elliptic crack, crack growth modeling, fatigue crack, fatigue damage accumulation.

DOI: 10.17516/1999-494X-2015-8-7-890-900.

(C) Siberian Federal University. All rights reserved

* Corresponding author E-mail address: ruslan239@mail.ru 


\title{
Прогнозирование развития
}

\section{четвертьэллиптической трещины усталости \\ с помощью конечно-элементного моделирования \\ накопления повреждений}

\author{
P.В. Гучинский ${ }^{\mathrm{a}, \tilde{\sigma}}$, C.В. Петинов ${ }^{\mathrm{a}, \bar{\sigma}}$ \\ ${ }^{a}$ Институт проблем машиноведения РАН \\ Россия, 199178, Санкт-Петербург, Большой проспект ВО, 61 \\ ${ }^{6}$ Санкт-Петербургский политехнический университет Петра Великого \\ Россия, 195251, Санкт-Петербург, Политехническая, 29
}

Предложен подход к оиенке развития пространственных трещин усталости, основанный на конечно-элементном моделировании накопления повреждений. Описываемая методика, использующая деформационный критерий разрушения и обобщенную ичиклическую кривую, обладает рядом преимуществ по сравнению с общепринятым форматом линейно-упругой механики разрушения. В соответствии с излагаемым подходом стадии развития трешиныне разделяются, рассматривается пластичность у вершины трещины, учитывается эффект раскрытия трещины, а также имеется возможность моделирования роста трещины от произвольного начального надреза или при его отсутствии. Использование подхода было проиллюстрировано на примере распространения четвертьэллиптической трещины от начального надреза. Результаты моделирования находятся в хорошем соответствии с экспериментальными данными.

Ключевые слова: четвертьэллиптическая трещина, усталость, моделирование роста трещин, накопление усталостных повреждений.

\section{1. Введение}

Болтовые и заклепочные соединения широко применяются в авиапромышленности и строительных сооружениях для механического скрепления элементов конструкций. Из-за податливости такие соединения имеют некоторое преимущество перед сварными соединениями при сопротивлении переменным нагрузкам и распространении трещин усталости. Проектирование болтовых и заклепочных соединений обычно осуществляется в рамках безопасного подхода, при этом подразумевается, что максимальное напряжение в области концентрации напряжений не превышает предела усталости и развития трещины не предполагается. Однако длительное время распространения трещины во многих случаях позволяет включить стадию ее развития, остаточный ресурс в общий ресурс конструкции. На этой идее основана концепция широко используемого принципа проектирования конструкций с допускаемым повреждением. Для оценки остаточного ресурса необходим анализ распространения трещины усталости. Усталостное разрушение заклепочных соединений начинается, как правило, с образования угловой четвертьэллиптической трещины в области концентрации напряжений у отверстия [1].

Оценка развития четвертьэллиптических трещин обычно выполняется в формате линейноупругой механики разрушения (ЛУМР) с помощью метода конечных элементов. Для двух основных наружных направлений роста трещины непрерывно оцениваются значения коэффи- 
циентов интенсивности напряжений (КИН) и производится интегрирование уравнения Пэриса [2]. Предполагается, что фронт сохраняет эллиптическую форму, что не всегда подтверждается экспериментом [3]. Однако Дж. Ньюмэн (J. Newman) и И. Райю (I. Raju) показали, что при измельчении сетки значения КИН в поверхностных точках резко уменьшаются [4], поэтому использование экстраполированного значения КИН для этих точек является не вполне обоснованным. Также экспериментальные данные демонстрируют, что константы уравнения Пэриса не могут считаться одинаковыми для двух направлений роста трещины [5]. Для устранения этого недостатка было предложено использование среднеквадратических КИН [6, 7], однако осреднение КИН по фронту трещины не совсем корректно из-за различия в напряженном состоянии и параметрах раскрытия трещины в его точках. Некоторые экспериментальные исследования показывают принципиальную невозможность использования КИН для оценки роста пространственной трещины [8].

Для анализа распространения полуэллиптической трещины также предлагался подход, основанный на оценке накопления повреждений и применении малоциклового критерия разрушения Коффина [9]. Расчет повреждения производился в двух основных направлениях ее роста. Однако в модели были использованы не только данные малоцикловых испытаний, но и интерполяционные формулы значений КИН для полуэллиптической трещины в растягиваемой пластине, полученные в [5].

В данной работе предложен подход к распространению двумерных трещин, основанный на конечно-элементном моделировании накопления повреждений и деформационном критерии разрушения. Подход обладает рядом преимуществ перед общепринятой методикой, основанной на ЛУМР. Во-первых, предоставляется возможность моделирования трещины от любого начального надреза при неустановившемся фронте трещины или даже при отсутствии исходной трещины. Поэтому начальная фаза распространения трещины и фаза устойчивого подрастания рассматриваются как единый процесс, управляемый постепенным накоплением повреждений. Во-вторых, использование деформационного критерия позволяет моделировать распространение трещины при развитой пластической деформации, что невозможно при использовании КИН. В-третьих, в предложенной модели принимается во внимание накопленное повреждение в пределах постоянно эволюционирующей области пластической деформации.

Целью настоящей работы является моделирование распространения угловой четвертьэллиптической трещины в алюминиевой пластине, развивающейся от начального надреза, и сопоставление результатов с экспериментальными данными.

\section{2. Методика моделирования накопления усталостных повреждений}

В соответствии с разработанным подходом область распространения трещины разбивается на конечные элементы одинаковых размеров. Предполагается, что эти «элементы материала» представляют собой некоторое число зерен с приблизительно одинаковой ориентацией плоскостей скольжения. Упрощенно поведение элемента материала перед вершиной трещины может быть представлено как деформирование образца при одноосном напряженном состоянии [10]. Процесс развития трещины усталости заключается в последовательном разрушении элементов материала в соответствии с законом накопления повреждений и выбранным критерием разрушения. Разрушение элемента материала моделируется путем искусственного уменьшения его 
жесткости на несколько порядков по сравнению с окружающим материалом. Соответственно, размер элемента является шагом приращения трещины в конкретной точке фронта. Моделирование трещины посредством удаления конечных элементов широко применяется при анализе хрупкого и квазихрупкого разрушения [11-13], гораздо реже - при знакопеременном нагружении для трещины усталости $[14,15]$.

Предполагается, что развитие повреждения не может происходить в течение части цикла, когда трещина закрыта. В связи с этим некоторая доля размаха деформации (нагрузки) оказывается «неэффективной» для развития разрушения. Для описания влияния неполного раскрытия трещины на процесс ее развития для каждого элемента вводится корректировка, параметр раскрытия $U$, идентичный предложенному Элбером [16]:

$$
\Delta \varepsilon_{e f f}=U \Delta \varepsilon
$$

В работе [17] для плоского напряженного состояния при КЭ-моделировании трещины бесконечно тонким разрезом был предложен способ оценки $U$. Предполагается, что раскрытие трещины происходит, когда знак узловой силы в вершине трещины становится положительным. При моделировании притупленной вершины трещины возможна оценка параметра раскрытия по изменению знака среднего напряжения в узлах элемента, лежащего у вершины трещины. Этот подход показал результаты, хорошо согласующиеся с экспериментальными данными для полуэллиптической трещины [18], и был использован в настоящей работе.

Упруго-пластическое поведение материала элементов описывается обобщенной циклической кривой, получаемой на основании стандартных испытаний образцов при одноосном напряженном состоянии. Как правило, результаты таких испытаний представляются в форме модифицированной аппроксимации Рэмберга-Осгуда [19]:

$$
\frac{\Delta \varepsilon}{2}=\frac{\Delta \sigma}{2 E}+\left(\frac{\Delta \sigma}{2 K^{\prime}}\right)^{1 / n^{\prime}},
$$

где $\Delta \varepsilon, \Delta \sigma$ - размахи деформаций и напряжений; $E$ - модуль Юнга; $K^{\prime}, n^{\prime}-$ коэффициенты упрочнения.

В качестве критерия разрушения элементов материала применяют деформационный критерий Мэнсона, учитывающий вклад в процесс разрушения упругой и пластической составляющей деформации. В соответствии с (1) в критерии используется эффективный размах полной деформации в направлении, перпендикулярном фронту трещины:

$$
\Delta \varepsilon_{\text {eff }} / 2=\left(\sigma_{f}^{\prime} / E\right)\left(2 N_{f}\right)^{b}+\varepsilon_{f}^{\prime}\left(2 N_{f}\right)^{c},
$$

где $E$ - модуль Юнга; $\sigma_{f}{ }_{f}$ и $\varepsilon_{f}{ }_{f}-$ коэффициенты усталостной прочности и пластичности; $b$ и $c-$ показатели степени; $N_{f}$ - количество перемен нагрузки (полуциклов) до разрушения образца, определяемое обычно по искажению «восходящей» части циклической кривой и резкому увеличению его податливости при возникновении трещины.

В данной работе исследуется распространение угловой четвертьэллиптической трещины в пластине из алюминиевого сплава 7075-Т651. Используемые параметры материала были получены при испытаниях в соответствии со стандартами ASTM Standards E8 and E606 [20]: n' = $0,074, K^{\prime}=852 \mathrm{M \Pi а,} E=70$ ГПа, $\sigma_{f}^{\prime}=1231 \mathrm{M \Pi а,} \varepsilon_{f}^{\prime}=0,26, b=-0,122, c=-0,806$. 
На начальном шаге расчета анализируется поле деформаций для всех элементов, лежащих в области распространения трещины, в которых развивается пластическая деформация. Эти элементы могут лежать в вершине начального выреза либо, при его отсутствии, в области концентрации напряжений, тогда в качестве индикатора начала разрушения может использоваться изначально введенная неоднородность в сопротивлении переменному нагружению. Затем с помощью деформационного критерия Мэнсона (3) устанавливается число циклов до разрушения каждого элемента $N_{i}$ и из них выбирается наименьшее значение $n_{1}$, соответствующее наиболее напряженному элементу. Для всех элементов вычисляется накопленное на начальном шаге повреждение, для наиболее напряженного элемента оно становится равным единице:

$$
d_{\text {initial }_{i}}=\frac{n_{1}}{N_{i}}
$$

После начального шага рассчитывается обновленное напряженно-деформированное состояние и определяется число циклов до разрушения следующего элемента с учетом накопленного им на начальном шаге повреждения. Когда трещина достигает определенного элемента, и повреждение становится критическим, он выключается из работы, перераспределяя усилия на окружающие его элементы. Значение повреждения элемента является суммой повреждений, накопленных им за все предшествующие циклы. Критерий разрушения каждого элемента описывается правилом линейного суммирования повреждений Пальмгрена-Майнера [21]:

$$
d_{i}=d_{\text {initial }_{i}}+\sum_{j} \frac{n_{j}}{N_{j}^{i}}=1
$$

где $n_{j}$ - число циклов до разрушения наиболее «слабого» элемента на $j$-м шаге подрастания трещины, определяемое его накопленным повреждением; $N^{i}$ - число циклов до разрушения $i$-го элемента на $j$-м шаге подрастания трещины, вычисляемое с помощью деформационного критерия разрушения (3).

Элементы описанной техники накопления повреждений основываются на ранних работах Глинки и Элайна $[22,23]$, которые получили свое развитие в работах $[10,24]$ и др. В этих исследованиях, однако, не использовался метод конечных элементов, поле деформаций у вершины трещины описывалось приближенно, а оценка роста трещины производилась только при плоском напряженном состоянии.

Разработанный подход может найти применение для анализа распространения пространственных трещин усталости, развивающихся от произвольного плоского надреза. Такой анализ необходим для оценок усталости и остаточного ресурса конструкций, работающих в условиях переменного нагружения (трубопроводы, сварные конструкции мостов [26], конструкции судов и морских сооружений [27] и др.).

\section{3. Моделирование развития четвертьэллиптической трещины}

Предложенный подход был использован для анализа развития четвертьэллиптических трещин, распространявшихся от начальных электроэрозионных надрезов. При проведении эксперимента образец в виде пластины из алюминиевого сплава 7075-Т651 со сквозным отверстием и начальными надрезами (рис. 1) испытывался при пульсирующем растяжении с мак- 
симальным номинальным напряжением 70 МПа и частотой 10 Гц [8]. Во время испытания несколько раз производилось снижение амплитуды нагрузки на 20 \% в течение нескольких сотен циклов для получения текущего контура фронта на берегах трещины. Такое незначительное изменение нагрузки, как показал эксперимент, не влияет на долговечность. Испытания проводились до достижения трещиной кромки пластины, противоположной начальному надрезу, для того чтобы избежать повреждения полученных отметок при возможном контакте берегов трещины.

В соответствии со схемой образца (рис. 1) была разработана конечно-элементная модель с достаточно мелкой сеткой в области предполагаемого развития трещины $(L=305$ мм, $W=$ $51 \mathrm{мм}, t=6,35 \mathrm{mм}, r=3,175 \mathrm{mм})$. Начальные надрезы размерами $1,2 \mathrm{x} 1,2$ мм моделировались

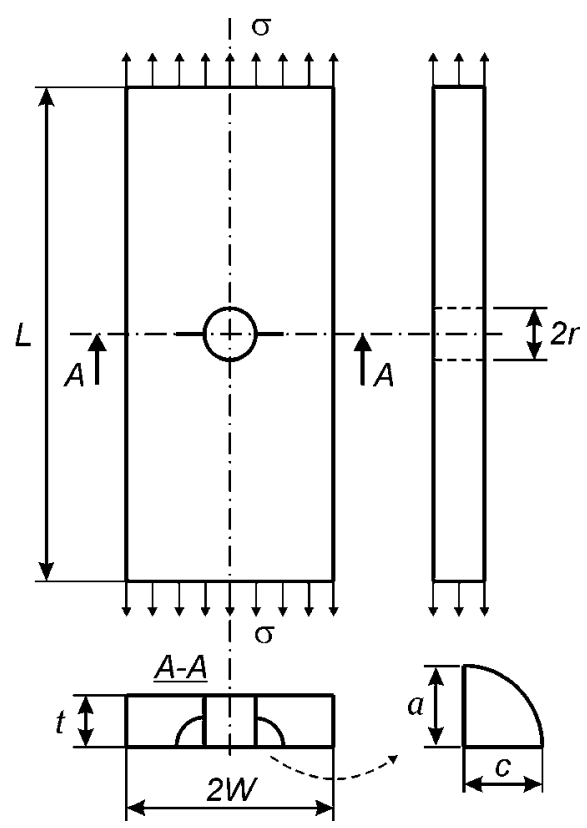

Рис. 1. Испытываемый образец с двумя угловыми четвертьэллиптическими трещинами



Рис. 2. Поле наибольших главных напряжений в окрестности вершины трещины: $a=3,5$ мм, $c=2,45$ мм 
с помощью присвоения малой жесткости элементам, лежащим в пределах надрезов. На каждом шаге подрастания трещины, т.е. после выхода из строя очередного элемента, анализировалось поле напряжений для определения параметра раскрытия трещины в каждой точке фронта (рис. 2), а также поле деформаций, перпендикулярных фронту трещины, для оценки числа циклов до разрушения каждого элемента. Когда повреждение элемента становилось равным единице, он выключался из работы в соответствии с (5), и производилась оценка обновленного напряженно-деформированного состояния. При таком пошаговом моделировании возможно отследить текущее положение контура фронта, состоящего из элементов малой жесткости для любого числа циклов. Например, на рис. 3 приведена последовательность девяти контуров фронта при значениях долговечности, составляющих $36,47,56,62,69,72,81,90,97$ \% от общей долговечности, характеризующейся выходом трещины на поверхность, противоположную начальному надрезу.

В работе [8] анализировалась эволюция фронта трещины в формате ЛУМР без учета влияния раскрытия трещины. Так как начальный надрез не отвечал дуге эллипса, расчет производился от трещины с примерно установившимся четвертьэллиптическим фронтом после первых нескольких тысяч циклов ( $c=1,7$ мм). Использовались данные по КИН, полученные в двух независимых исследованиях. Результаты оценки распространения трещины нельзя признать одновременно удовлетворительными по фронту трещины (рис. 4) и долговечности. Число циклов, необходимое для развития установившейся трещины до ее выхода на внешнюю поверхность, вычисленное с использованием КИН, полученных с использованием сингулярных элементов (Newman and Raju, 21534 цикла), переоценивает экспериментальное число циклов (13470), в то время как по фронту наблюдается хорошее согласование. Напротив, при более выраженном соответствии по числу циклов (9610) фронт трещины, полученный с помощью КИН на основании техники экстраполяции STRIPE, слабо согласуется с экспериментальными результатами [8].

В данной работе была выполнена оценка роста трещины с помощью техники накопления повреждений от начального надреза. Преимущество этого метода состоит в возможности моделирования трещины от любого начального надреза. Число циклов, в течение которых про-

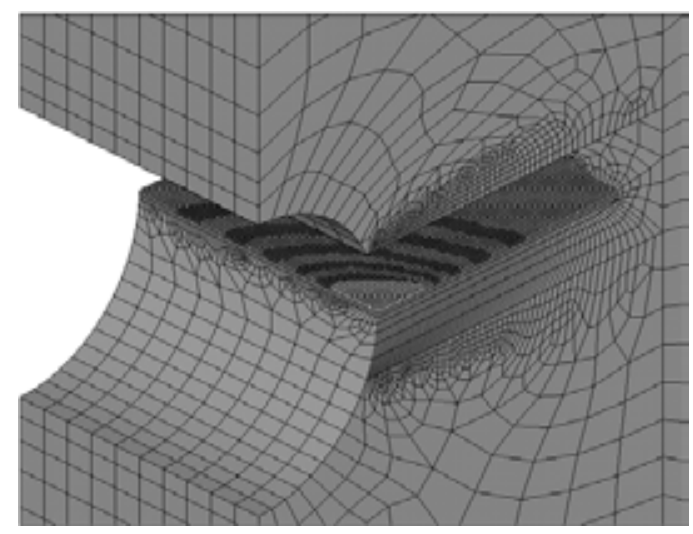

Рис. 3. Эволюция контура фронта трещины, полученная с помощью моделирования накопления повреждений 


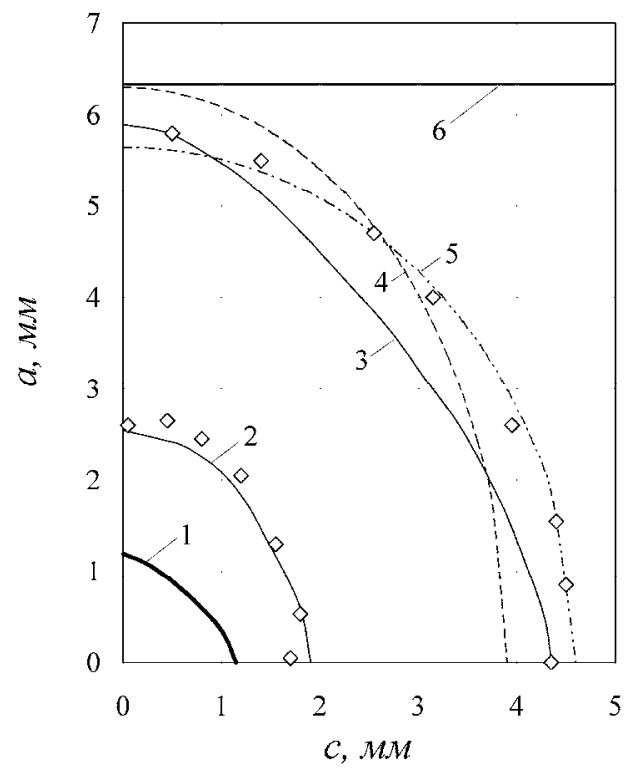

Рис. 4. Сопоставление оценок фронта трещины, развивающейся от начального надреза 1, полученных: 2, 3 - моделированием накопления повреждений, 4 - в формате ЛУМР техникой STRIPE [8], 5 - в формате ЛУМР с использованием сингулярных элементов (Newman and Raju, [8]); 6 - кромка пластины. Маркеры - экспериментальные данные [8]

исходило подрастание трещины между двумя контрольными отметками, оказалось меньше экспериментального значения (10930), а фронт немного менее искривленным (рис. 4). Изменение ширины трещины при ее росте удовлетворительно согласуется с экспериментальными данными (рис. 5).

Известно, что остаточные напряжения ввиду своей неоднородности могут значительно влиять на конфигурацию фронта трещины, часто вызывают искривление фронта эллиптических трещин у поверхности $[3,25]$. Данные эксперимента показывают, что при подрастании рассматриваемой трещины наблюдалось замедление ее роста в поверхностных точках (рис. 4). Причем по мере роста влияние остаточных напряжений уменьшалось из-за их постепенной релаксации. В некоторых образцах происходило полное искажение четверть-эллиптического фронта [8], обязанное, очевидно, наличию остаточных сжимающих напряжений, образовавшихся после изготовления образцов. При конечно-элементном анализе тормозящее влияние остаточных напряжений у поверхности трещины не было учтено, поэтому скорость ее развития оказалась больше экспериментальной, трещина достигла противоположной поверхности при меньшем числе циклов (рис. 5), а фронт оказался более пологим. Учет роли остаточных напряжений может позволить получить корректный прогноз фронта четвертьэллиптической трещины.

Анализ раскрытия трещины для различных точек фронта выявил, что параметры раскрытия принимают значения, мало отличающееся от единицы, по-видимому, из-за пластических свойств высокопрочного алюминиевого сплава. Поэтому трещина оказалась практически полностью раскрытой во время подрастания, и оценки ее развития в рамках ЛУМР удовлетворительно согласуются с экспериментальными данными без учета эффекта раскрытия.

$$
-897-
$$




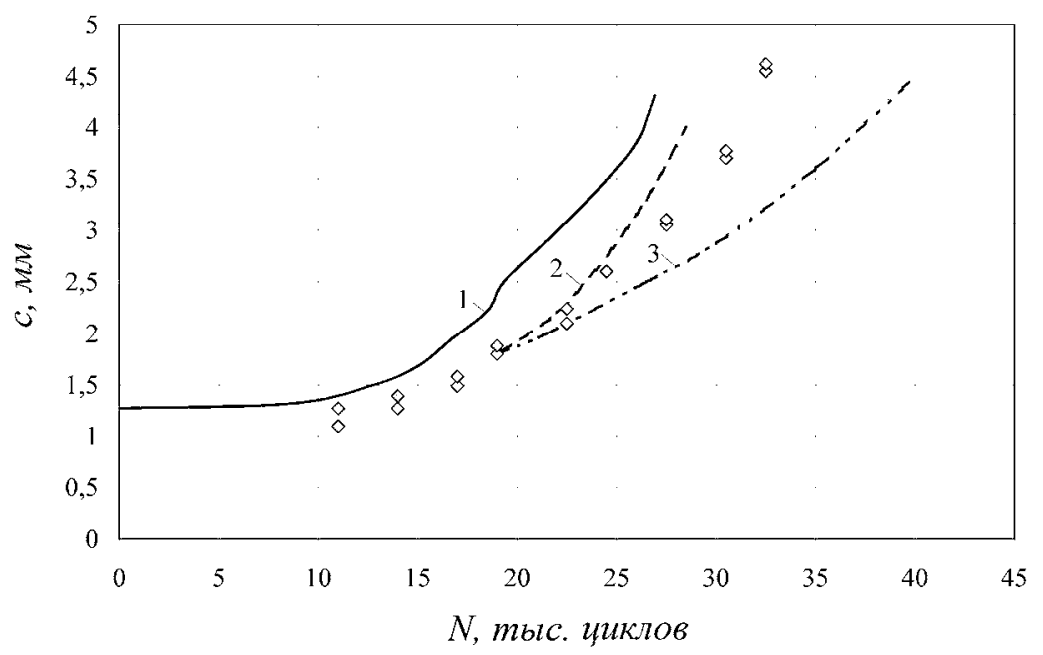

Рис. 5. Зависимость ширины трещины от числа циклов, полученная: 1 - моделированием накопления повреждений, 2 - в формате ЛУМР техникой STRIPE [8], 3 - в формате ЛУМР с использованием сингулярных элементов (Newman and Raju, [8]). Маркеры - экспериментальные данные для двух симметричных трещин [8]

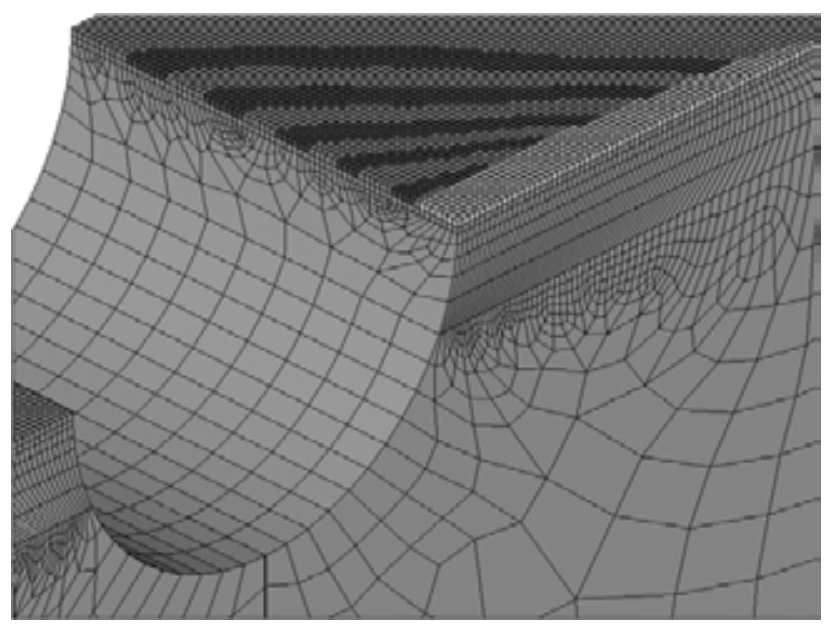

Рис. 6. Эволюция фронта трещины при начальном широком надрезе

В одном из образцов с начальным протяженным надрезом (рис. 6, светлые элементы) эксперимент показал невозможность использования КИН для анализа развития трещины. Формат ЛУМР предполагает равномерное развитие трещины от такого надреза по всем направлениям с разной интенсивностью. Однако в данном случае трещина начинает рост от нового дефекта на контуре надреза и ее дальнейшее развитие малочувствительно к его начальной форме. Использование техники накопления повреждений позволяет моделировать такое поведение трещины. Получаемая в результате моделирования эволюция контура фронта удовлетворительно согласуется с экспериментальными данными (рис. 6). Неучет торможения в поверхностном слое из-за наличия остаточных напряжений приводит к более пологой форме фронта по срав- 
нению с наблюдаемой в эксперименте. Количественная оценка остаточных напряжений и разработка подходов к моделированию их воздействия является возможным направлением для дальнейшей работы.

\section{4. Заключение}

В работе выполнено моделирование развития угловых четвертьэллиптических трещин усталости от начального дефекта. Для этого был выбран подход, основанный на конечноэлементном моделировании накопления повреждений. В предлагаемом подходе используется деформационный критерий разрушения и обобщенная циклическая кривая, которые можно получить в результате испытаний при одноосном напряженном состоянии, а также учитывается эффект раскрытия трещины. В сравнении с общепринятой методикой оценки развития пространственных тещин усталости, основанной на аппарате ЛУМР, представленный подход обладает рядом преимуществ. Во-первых, его применение не требует определенной формы начального надреза и возможно даже при его отсутствии. Поэтому подход не разделяет фазы образования и развития трещины, рассматривая их в рамках единого процесса. Во-вторых, учитывается пластическая деформация, тесно связанная с процессом накопления необратимых повреждений. В-третьих, имеется возможность моделирования трещины при больших пластических деформациях, когда исчезает сингулярность напряжений у вершины трещины и применение ЛУМР становится невозможным. Наконец, непрерывный анализ повреждения элементов материала позволяет учесть повреждение, накопленное в пределах пластической области во время предшествующего нагружения. Рассматриваемый подход позволяет сохранить информацию о поврежденности материала, которая теряется при перестроении сетки для анализа в рамках ЛУМР.

Результаты моделирования четвертьэллиптической трещины от нескольких начальных надрезов показали хорошую согласованность с экспериментальными данными. Незначительные (консервативные) отклонения от результатов эксперимента могут быть объяснены тем, что не учитывались остаточные напряжения.

\section{Список литературы}

[1] Shin C.S. // Int. J. Fatigue. 1991. Vol. 13. P. 233-240.

[2] Lin X.B., Smith R.A. // Engineering Fracture Mechanics. 1998. Vol. 59. P. 73-87.

[3] Fleck N.A., Smith I.F.C., Smith, R.A. // Fatigue \& Fracture of Engineering Materials \& Structures. 1983. Vol. 6. P. 225-239.

[4] Newman J.C.Jr., Raju I.S. // Stress-Intensity factor equations for cracks in three-dimensional finite bodies, NASA TM 83200 Langley Research Center Hampton VA. 1981. P. 54.

[5] Raju I.S., Newman J.C.Jr. // Analyses of surface cracks in finite plates under tension or bending loads, NASA TP-1578. 1979. P. 46.

[6] Chahardehi A., Brennan F.P., Han S.K. // Int. J. Fatigue. 2010. Vol. 32. P. 297-301.

[7] Brennan F.P., Ngiam S.S., Lee C.W. // Engineering Fracture Mechanics. 2008. Vol. 75. N. 3-4. P. 355-363.

[8] Fawaz S.A., Andersson B., Newman J.C.Jr. // Proc. of the 22nd Symposium of the International Committee on Aeronautical Fatigue. Lucerne. 2003. P. 31. 
[9] Shi K., Cai L., Chen L., Bao C. // Chin. J. Aeronaut. 2014. Vol. 27. N 3. P. 730-734.

[10] Castro J.T.P., Meggiolaro M.A., Miranda A.C.O. // Computational Materials Science. 2009. Vol. 46. P. 115-123.

[11] Unossona M., Olovssona L., Simonsson K. // Elements in Analysis and Design. 2006. Vol. 42. P. 283-297.

[12] Fan R., Fish J. // International Journal for Numerical Methods in Engineering. 2008. Vol. 73. P. $1607-1623$.

[13] Капустин С.A // Проблемы прочности и пластичности: Межвуз. сборник. Вып. 71. Н. Новгород, 2009. С. 227.

[14] Королев И.К., Петинов С.В., Фрейдин А.Б. // Вычислительная механика сплошных сред. 2009. Т. 2. Вып. 3. С.34-43.

[15] Peerlings R.H.J., Brekelmans W.A.M., Borst R., Geers M.G.D. // Int. J. Numer. Meth. Engng. 2000. Vol. 49. P. 1547-1569.

[16] Elber W. // Eng. Fracture Mech. 1970. Vol. 2. P. 37-45.

[17] Wu J., Ellyin F. // International Journal of Fracture. 1996. Vol. 82. P. 43-65.

[18] Guchinsky R. V., Petinov S. V. // Proceedings of XLII International Summer SchoolConference APM 2014. St-Petersburg, Repino, 2014. P. 301-311.

[19] Petinov S.V. Fatigue Analysis of Ship Structures. Backbone Publishing Co. 2003, P.O. Box 562 Fair Lawn NJ 07410. P. 262.

[20] Fatemi A., Plaseied A.K., Khosrovaneh D. // Int. J. Fatigue. 2005. Vol. 27. P. 1040-1050.

[21] Miner M.A. // Journal of Applied Mechanics. 1945. Vol. 67. P. 159-165.

[22] Glinka G. // Int. Journal of Fatigue. 1982. Vol. 4. N 2. P. 59-67.

[23] Ellyin F., Fakinlede C.O. // Engineering Fracture Mechanics. 1985. Vol. 22. N 4. P. 697-712.

[24] Chen L., Cai L.X., Yao D. // Chin. J. Aeronaut. 2013. Vol. 26. N 1. P. 130-135.

[25] Fawaz S.A. Fatigue crack growth in riveted joints Ph.D. Thesis Delft University of Technology Faculty of Aerospace Engineering. 1997. P. 230.

[26] Guchinsky R.V., Petinov S.V. // Инженерно-строительный журнал. 2013. Т. 1. Вып. 36. C. 39-47.

[27] Petinov S.V., Guchinsky R.V. // Transactions of the Royal Institution of Naval Architects. International Journal of Maritime Engineering. 2013. Vol. 155. Part A4. P. 201-209. 